\title{
Comment on: Vulvar basal cell carcinoma: clinical features and treatment outcomes from a tertiary care centre
}

Singapore Med J 2020; 61(3): 167 https://doi.org/10.11622/smedj.2020032

Dear Sir,

We read with great interest the paper by Namuduri et al regarding the clinical behaviour and management of vulvar basal cell carcinoma. ${ }^{(1)}$ We feel that the paper is lacking with regard to discussing the diagnosis of these insidious lesions, as it made no mention of dermatoscopy. Dermatoscopy is an in vivo non-invasive technique that permits visualisation of numerous morphologic features invisible to the naked eye, thus enhancing the clinical diagnosis of nearly all pigmented and non-pigmented mucosal lesions. ${ }^{(2)}$ As mentioned by Namuduri et al, symptoms of vulvar melanoma include pain, itching and bleeding, all of which are signs of a late diagnosis. If we really want to improve the diagnosis and the prognosis of basal cell carcinoma in this particular site, we need to arrive at a true early diagnosis that can only be achieved with the use of new non-invasive diagnostic techniques in the vulvar area, which have already been successfully used on the skin. In fact, thanks to dermatoscopy, we are able to discriminate between a melanocytic and non-melanocytic lesion with the recognition of particular dermatoscopic parameters and various colours that we find within the lesion. Dermatoscopy allows us to do this with better patient management and reach considerably greater diagnostic accuracy that just using the naked eye.

Therefore, in our experience, the addition of dermatoscopy to routine screening for mucosal vulvar lesions is clinically relevant. The use of dermatoscopy not only enables early diagnosis of basal cell carcinoma, but also allows more appropriate classification of lesions that appear suspicious to the naked eye, thereby reducing false positive findings on clinical examination and ultimately reducing the number of superfluous surgical excisional biopsies..$^{(3-5)}$

Dermatoscopy enables non-invasive diagnoses without subjecting the patient to unpleasant excisions of the vulvar area. It is essential that these non-invasive diagnostic methods are disseminated to and known by all doctors who manage these lesions, whether they are gynaecologists or dermatologists, and used routinely during normal patient visits. Only then can we obtain early diagnoses of patients with vulvar basal cell carcinoma, avoiding vulvectomies, which are extremely invalidating for our patients, both from a psychological and functional point of view.

Consequently, in the light of the strong clinical evidence and numerous indexed papers in the past few years, we argue that dermatoscopy should at least have been mentioned in Namaduri et al's paper.

Yours sincerely,

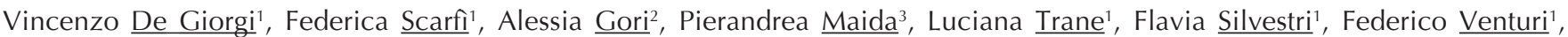
Piero Covarelli ${ }^{4}$

${ }^{1}$ Department of Dermatology, University of Florence, Florence, ${ }^{2}$ Cancer Research 'Attilia Pofferi' Foundation, Pistoia, Italy, ${ }^{3}$ Clinica Luganese Moncucco, Lugano, Switzerland, ${ }^{4}$ Department of Surgery, University of Perugia, Perugia, Italy. vincenzo.degiorgi@unifi.it

\section{References}

1. Namuduri RP, Lim TY, Yam PK, et al. Vulvar basal cell carcinoma: clinical features and treatment outcomes from a tertiary care centre. Singapore Med J 2019; 60: 479-82.

2. Argenziano G, Soyer HP. Dermoscopy of pigmented skin lesions--a valuable tool for early diagnosis of melanoma. Lancet Oncol 2001; 2:443-9.

3. de Giorgi V, Massi D, Salvini C, et al. Thin melanoma of the vulva: a clinical, dermoscopic-pathologic case study. Arch Dermatol 2005; 141:1046-7.

4. de Giorgi V, Massi D, Mannone F, et al. Dermoscopy in vulvar basal cell carcinoma. Arch Dermatol 2007; 143:426-7.

5. De Giorgi V, Massi D, Lotti T. Basal-cell carcinoma. N Engl J Med 2006; 354:769-71. 